\title{
Ce que dire que l'on parle veut dire : réflexion sur les compétences linguistiques des derniers
}

\section{Ottomans}

To speak or not to speak: some considerations on language proficiency in the late Ottoman period

\section{Olivier Bouquet}

\section{(2) OpenEdition}

\section{Journals}

Édition électronique

URL : http://journals.openedition.org/ejts/1523

DOI : 10.4000/ejts. 1523

ISSN : 1773-0546

Éditeur

EJTS

Référence électronique

Olivier Bouquet, «Ce que dire que l'on parle veut dire : réflexion sur les compétences linguistiques des derniers Ottomans », European Journal of Turkish Studies [En ligne], 6 | 2007, mis en ligne le 05 mars 2015, consulté le 16 février 2020. URL : http://journals.openedition.org/ejts/1523 ; DOI : 10.4000/ejts. 1523 


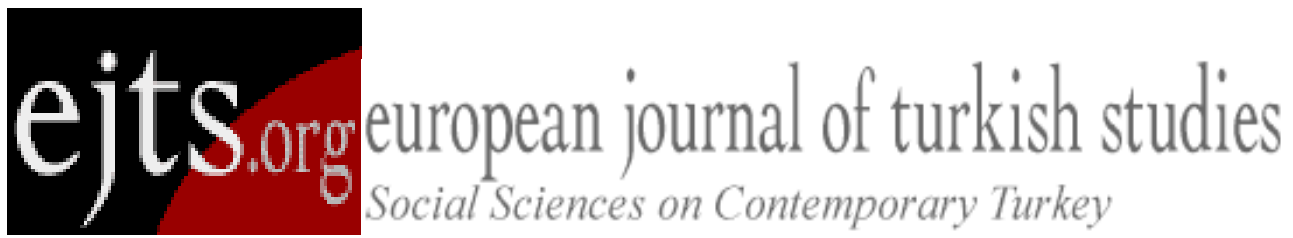

Citation : Bouquet, Olivier (2007) 'Ce que dire que l'on parle veut dire : réflexion sur les compétences linguistiques des derniers Ottomans', European Journal of Turkish Studies, Thematic Issue N6, III-literate Knowledge, URL: http://www.eits.org/document1523.html

To quote a passage, use paragraph (§).

\title{
Ce que dire que l'on parle veut dire : réflexion sur les compétences linguistiques des derniers Ottomans
}

Olivier Bouquet

\begin{abstract}
To speak or not to speak: some considerations on language proficiency in the late Ottoman period'. - Language knowledge has often been used as a double dividing line between written and oral cultures, and between traditional skills (the three languages of the adab culture) and modernist dispositions (related with European languages, mainly French). However, a precise study of the terminology of the biographical registers set up during Abdülhamid the Second's rule (1876-1909) by the Commission of Personnel Records (sicill-i ahvâl komisyonu) reveals that this interpretative framework is flawed, partly due to the nature of selfassessment practices. Indeed, some employees did not always mention the languages they could effectively use; their colleagues who did so sometimes declared written abilities while omitting oral ones; conversely, those who sought to secure or promote their careers would claim knowledge of French even if they could not speak a single word of it. Although the Commission was aware of these problems and tried to implement normative coding procedures, this affected biographical discourses in particular ways. As a result, if one addresses the question of literacy as more related to practical capacities than cultural orientations or theoretical knowledge, one draws a collective portrait different from the individual analysis of self-evaluations and reputations: on the one hand, the cultivated modernist elites do not always display remarkable skills; on the other hand, some employees who were versed in a limited number of languages and had poor linguistic reputations had often a more sophisticated relation with literacy than what appears in the sources.
\end{abstract}


Citation : Bouquet, Olivier (2007) 'Ce que dire que l'on parle veut dire : réflexion sur les compétences linguistiques des derniers Ottomans', European Journal of Turkish Studies, Thematic Issue $\mathrm{N}^{\circ} 6$, III-literate Knowledge, URL: http://www.ejts.org/document1523.html

To quote a passage, use paragraph (§).

Les historiens ottomanistes qui abordent l'usage des langues à la fin de l'Empire ottoman se répartissent en deux catégories. Les premiers envisagent le rapport aux langues comme l'objet d'un savoir (qui sait le turc, qui sait l'arabe) ou le résultat d'un apprentissage rapporté à un moment biographique (qui l'a appris à l'école, en famille, ou en bureau). Les seconds distinguent la fonction d'oralité de la fonction écrite, liées tantôt à la nature de la langue (le kurde se parle plus qu'il ne s'écrit), tantôt à son lieu de pratique (l'albanais se parle dans les parties albanaises de l'Empire) ou encore à la population locutrice considérée (un gouverneur ottoman dans une province arabe s'adresse en turc à sa hiérarchie). Or le premier angle néglige de prendre en compte qu'une langue est aussi - voire avant tout - objet de compétence comme le disent les linguistes, et que cette compétence est évolutive. II est ainsi problématique de considérer qu'un Ottoman qui dit ne pas savoir une langue est illettré ou muet dans cette langue - en réalité il peut la parler ou l'écrire, mais estime ne pas être en situation de rendre compte de cette aptitude. Pour ce qui est du second angle d'approche, il néglige les modalités du discours sur la langue: si un Ottoman dit qu'il écrit une langue, c'est peut-être qu'il ne fait que l'écrire ; c'est peut-être au contraire qu'il la parle, mais pas assez bien à ses yeux pour faire état de ce savoir parler.

[2] À partir du discours tenu par les pachas hamidiens sur leur pratique des langues, on discutera ces deux approches. On verra que le silence qui entoure le savoir linguistique dans les sources biographiques ne dit pas tout de la réalité de trois savoirs imbriqués (savoir parler, lire ou écrire), en ce sens qu'il relève de l'autoportrait linguistique tel que l'envisage le sujet qui parle de lui. Cela nous permettra de montrer que l'opposition habituellement tracée par les ottomanistes entre culture orale et culture écrite doit être complétée, voire nuancée, par la prise en compte, à échelle individuelle, des niveaux de maîtrise et des degrés de compétence : la seule confrontation de deux types idéaux (le sage illettré contre l'érudit muet) peut être enrichie par l'identification d'un savoir linguistique (dans le sens de literacy tel qu'évoqué en introduction du dossier) exprimé sous mille formes, du lecteur non scripteur, au polyglotte qui parle toutes les langues mais en turc. 
Citation : Bouquet, Olivier (2007) 'Ce que dire que l'on parle veut dire : réflexion sur les compétences linguistiques des derniers Ottomans', European Journal of Turkish Studies, Thematic Issue $\mathrm{N}^{\circ} 6$, III-literate Knowledge, URL: http://www.ejts.org/document1523.html

To quote a passage, use paragraph (§).

\section{Dire ce que l'on sait}

[3] L'enquête repose sur l'exploitation de 282 notices biographiques individuelles enregistrées durant le règne du sultan Abdülhamid II (1876-1909). On y trouve de précieuses informations sur les compétences linguistiques des pachas. Si la Commission des sicill-i ahvâl (instituée en 1879 afin de répertorier les notices de l'ensemble des employés de l'administration civile) exige de ces hauts dignitaires de telles informations, c'est que la maîtrise des langues oriente la décision d'attribution des postes, notamment dans les provinces : un décret d'octobre 1891 stipule par exemple que la connaissance de l'arabe est nécessaire à une nomination au poste de directeur de la correspondance du vilâyet de Beyrouth, afin de pouvoir contrôler les publications journalistiques locales; Ibrahim Fevzi est nommé gouverneur du Necd (Najd), entre autres parce qu'il connaît l'arabe ; Abdülvahhab est nommé gouverneur de Mytilène car il 'connaît la langue', c'est-à-dire le grec; à l'inverse, le supérieur hiérarchique d'Abdullah Ragıb, à savoir Zihni Paşa, gouverneur général de Crète, trouve dans le fait que son subordonné 'ignore la langue' - je suppose qu'il s'agit du grec qu'Abdullah ne cite pas comme langue pratiquée - le motif de son renvoi du poste de gouverneur de Kandiye (La Candie). Bref, le pouvoir prend bel et bien en compte les capacités linguistiques des employés pour mieux les mobiliser face à 'l'enjeu des provinces' (F. Georgeon). Ceci une fois établi, le problème pour l'historien est de mesurer la valeur du portrait que ces employés livrent d'eux-mêmes. Pour ce faire, partons de la comparaison de deux pachas qui font état des mêmes langues pratiquées, mais différemment :

\begin{tabular}{|l|l|l|l|}
\hline & Turc & Arabe & Persan \\
\hline Ahmed İzzet & Parle et écrit & Parle et écrit & Parle et écrit \\
\hline Hüseyin Rüşdü & Écrit & Parle & Parle \\
\hline
\end{tabular}

[4] Ces deux pachas disent parler et (ou) écrire les mêmes langues. En ce sens, leurs indications satisfont exactement à la lettre des règlements impériaux : ce qui intéresse l'administration centrale porte davantage sur des aptitudes (savoir parler ou écrire) que sur l'existence d'un savoir linguistique. En l'espèce, alors que le terme fréquemment employé kitabet désigne la maîtrise de l'écriture (yazı yazmak), de la composition littéraire ; étymologiquement autant que pratiquement, il renvoie à l'art du kâtib, à la possibilité d'accomplir toutes les fonctions d'écriture d'un bureau. Quant au terme tekellüm, il implique une capacité à échanger aussi bien avec les gens 
Citation : Bouquet, Olivier (2007) 'Ce que dire que l'on parle veut dire : réflexion sur les compétences linguistiques des derniers Ottomans', European Journal of Turkish Studies, Thematic Issue $\mathrm{N}^{\circ} 6$, III-literate Knowledge, URL: http://www.ejts.org/document1523.html

To quote a passage, use paragraph (§).

de bureau qu'avec les administrés. Or c'est de cette double aptitude fort différente que la Commission des sicill-i ahvâl tient compte dans l'optique du processus de nomination ou de révocation. Dans l'un des documents préparatoires à la création de cette administration, il est en effet précisé que si la connaissance se limite aux bases grammaticales (usul) et au vocabulaire (lugât) ordinaires, l'employé devra écrire : 'je n'écris ni ne parle mais en suis familier (aşina)' ; dans l'autre cas, il devra noter : 'j'écris et je parle'. C'est précisément dans ce cas que se place Hüseyin Şükrü, qui sous-entend que les notions qu'il possède ne lui permettent pas d'écrire ou de parler.

\begin{tabular}{|l|l|l|l|}
\hline & Turc & Arabe & Persan \\
\hline Hüseyin Şükrü & Écrit & Familier & Familier \\
\hline
\end{tabular}

[5] À l'évidence, le dispositif lexical de l'administration s'impose : la majorité des pachas y recourent. Mais une partie d'entre eux le contournent, préférant employer d'autres termes qui renvoient tantôt à l'écrire (yazar, tahrir), tantôt au parler (söyler). De telles nuances ne sont pas seulement rhétoriques: dans un monde où les différences de maîtrise sont considérables, le dignitaire tient à expliciter un aspect de son rapport à la langue, qu'il s'agisse de sa capacité linguistique générale: la compréhension (tefehhüm, istihrac) ou la connaissance (bilir, vakıf) ; qu'il s'agisse d'un rapport particulier au texte, comme la lecture (krraat, okur), plus souvent associée à l'écriture qu'à la traduction (tercüme), la poésie (şiir) ou la composition (inşa).

\section{Selon que l'on est humble ou prétentieux}

[6] Un pacha dit savoir ou pratiquer telle ou telle langue. Dans la plupart des cas, on peut penser qu'il dit vrai : le monde des pachas est petit; la connaissance des langues est un trait distinctif, un fait de réputation; elle est symboliquement si forte, si ségrégative socialement, que l'administration aura toujours la possibilité de s'informer, lorsqu'elle aura à nommer quelqu'un dont elle voudra être certaine qu'il parle telle langue au motif que la fonction l'exige. II n'est pourtant pas à exclure que certains ne sachent presque rien des langues qu'ils citent: Ahmed Hamdi dit parler arabe ; un consul britannique tient qu'il l'ignore. D'autres à l'inverse négligent de mentionner une langue, comme s'il était implicite qu'ils la connaissent, surtout dans le cas du turc. Ils préfèrent davantage citer des langues plus distinctives, dont il ne va absolument pas de soi qu'ils les parlent ou 
Citation : Bouquet, Olivier (2007) 'Ce que dire que l'on parle veut dire : réflexion sur les compétences linguistiques des derniers Ottomans', European Journal of Turkish Studies, Thematic Issue $\mathrm{N}^{\circ} 6$, III-literate Knowledge, URL: http://www.ejts.org/document1523.html

To quote a passage, use paragraph (§).

les écrivent. Ainsi Mehmed Said Halim n'indique que deux langues, l'arabe et le turc (kitabet). II a pourtant étudié le persan, le français et l'anglais, avec des instructeurs ; il est de la famille du khédive où les polyglottes sont monnaie courante ; on dit de lui qu'il connaît 'plusieurs langues' ; il a fait des études en Suisse pendant cinq ans, tout comme ses frères Mehmed Ali Halim et Ibrahim Halim qui, précisément, disent parler le français et l'anglais ; il a écrit en français. Bref, à moins que Mehmed Said Halim considère que sa mention d'étude linguistique suffit à suggérer son niveau de langue mais alors pourquoi en indique-t-il certaines plutôt que d'autres ? -, il estime que le turc et l'arabe sont les seules qui feraient doute aux yeux de l'administration, la maîtrise de l'anglais et du français étant impliquée par son origine familiale et sa biographie scolaire, alors que le turc ou l'arabe ne sont peut-être pas parfaitement maîtrisés par les membres de la famille, notamment ceux qui vivent en Égypte ou en Europe. J'en veux pour preuve que son frère Mehmed Ali Halim ne cite pas ces langues et que son autre frère İbrahim Halim ne fait pas mention de l'arabe.

[7] Cas de figure inverse : le pacha passe sous silence des langues par ailleurs connues, dont il ne va pas de soi qu'il les sache, soit qu'il estime ne pas avoir à répéter les langues déjà mentionnées dans la section de sa fiche biographique qui porte sur la scolarité ; soit qu'il considère que son niveau n'est pas assez élevé pour qu'il en fasse mention : à en croire Ali, celui-ci ne 'lit et écrit' que le turc et le français, alors que l'historiographe Mehmed Süreyya affirme qu'il est 'familier des langues'. De même, le grand vizir Ahmed Cevad cite trois langues: le turc (kitabet), le français (tekellüm) et l'arabe (aşina). Or nous savons qu'il en connaît d'autres : le persan, le grec et l'italien. Son silence en l'espèce n'a d'égal que le sérieux qu'il met dans l'application des règlements ou que la modestie dont il fait montre - il estime peut-être ne pas connaître assez bien ces langues pour les citer.

[8] À l'en croire, il n'aurait que des notions d'arabe. II en sait pourtant bien plus : il vient d'une grande famille d'oulémas et de professeurs d'arabe, une langue que son père connaissait bien. Şerif Ahmed Rauf 'parle' français ; pour avoir passé deux années dans un internat français, il doit bien l'écrire un peu. II y a là comme une modestie placée là où d'autres font montre de vantardise : Receb Hifzı dit écrire le turc, parler l'arabe, l'albanais, le grec, le serbe, le bulgare, et comprendre le français, le russe et l'italien; on ne peux pas jeter le soupçon sur son discours sous prétexte que le pacha n'a pas étudié au-delà de l'école secondaire rüşdiye (dont curieusement il a perdu le diplôme), 
Citation : Bouquet, Olivier (2007) 'Ce que dire que l'on parle veut dire : réflexion sur les compétences linguistiques des derniers Ottomans', European Journal of Turkish Studies, Thematic Issue $\mathrm{N}^{\circ} 6$, III-literate Knowledge, URL: http://www.ejts.org/document1523.html

To quote a passage, use paragraph (§).

qu'il n'a pas bénéficié de professeurs particuliers, et qu'il s'est peu déplacé dans des régions où il aurait pu pratiquer ces langues; mais on aurait bien aimé l'entendre exprimer les qualités qu'il se prête.

[9] J'ajoute qu'un niveau de langue est souvent cité relativement à un autre. Si la Commission des registres définit normativement le sens d'aşina (comme familiarité avec une langue, mais incapacité à l'écrire ou à la parler), c'est que chacun interprète ce terme à la lumière de sa perception de la réalité socio-linguistique ottomane. Ainsi, Ahmed Cevdet laisse-t-il entendre qu'il maîtrise pleinement l'arabe et le persan, ce dont personne ne saurait douter tant sa réputation en sciences islamiques et en poésie mesnevi est établie. En comparaison, son jugement est bien plus mesuré lorsqu'il évoque sa pratique du français. Une telle prudence n'a rien d'étonnant : sa fille, Fatma Aliye, rapporte qu'il ne s'est jamais senti à l'aise quand il s'agissait de parler cette langue. Sans doute des collègues peu bienveillants l'ont-ils noté pour en tirer parti à la première occasion. Midhat Paşa, qui ne l'apprécie guère, ne se prive pas de le faire lors des débats sur le projet constitutionnel de 1876: il sous-entend publiquement qu'Ahmed Cevdet n'est assez équipé en français pour comprendre les subtilités du droit européen. Seulement, Ahmed Cevdet connaît également son adversaire, et lui rend la monnaie de sa pièce lorsqu'il lui rétorque que Midhat n'est pas le mieux placé pour lui faire la leçon sur ce chapitre. Ahmed Cevdet sait qu'il lit bien le français, et en particulier celui des institutions et du droit. Cela dit, il sait tout autant qu'il n'arrive pas à la cheville des plus brillants, qu'il n'atteindra jamais le niveau de son très regretté maître, Mustafa Reşid Paşa, styliste hors pair s'il en fût. Dans sa fiche biographique, Ahmed Cevdet est donc modeste, avec raison.

\section{L'acquisition des compétences : biographies linguistiques}

[10] L'homme de langue acquiert progressivement une compétence, laquelle est soumise à l'ordre des acquisitions et des pertes. Huseyin Tevfik dit dans sa fiche qu'il parle anglais. La connaissance qu'il a de cette langue est alors celle d'un ambassadeur en poste aux États-Unis. Certes, Tahir Paşa, diplômé de Cambridge, s'est occupé de lui ; certes, il a effectué avant cette ambassade une mission aux États-Unis. Mais si la notice avait été enregistrée vingt ans auparavant, aurait-il indiqué ce savoir parler ? II est tout aussi probable que, ne pratiquant plus cette langue après 
Citation : Bouquet, Olivier (2007) 'Ce que dire que l'on parle veut dire : réflexion sur les compétences linguistiques des derniers Ottomans', European Journal of Turkish Studies, Thematic Issue $\mathrm{N}^{\circ} 6$, III-literate Knowledge, URL: http://www.ejts.org/document1523.html

To quote a passage, use paragraph (§).

son retour de mission l'année suivante, il l'oublie progressivement, au point de ne plus la parler dix ans plus tard. Bref, sa connaissance linguistique s'inscrit dans une trajectoire qu'il convient de reconstituer. Pour explorer les possibilités de l'exercice, je traiterai d'un cas bien documenté, celui d'Ahmed Cevdet.

[11] À la date de l'enregistrement de sa fiche, le 20 juin 1879, voici ce qui est indiqué : 'Il parle et écrit l'arabe et le persan ; s'il a des connaissances en français et en bulgare, il ne parle pas ces langues'. Partons d'un constat: il manque une langue, le turc. Ainsi que je l'ai signalé pour d'autres pachas, Cevdet considère probablement qu'il va de soi qu'il la sache, et néglige de la mentionner. Mais nous savons que c'est la langue de sa famille, et donc celle qu'il a apprise en premier, celle qu'il connaît le mieux. II commence à la parler dès son plus jeune âge, à l'écrire à l'école de quartier quelques années après, à la maîtriser de mieux en mieux les années et les décennies suivantes. Dans sa première jeunesse également, il se frotte à deux autres langues : au bulgare, langue parlée dans la ville mixte qu'est Lofça ; il l'entend hors de chez lui, dans la rue ou ailleurs. Mais comme il n'est pas Pomak, il est probable qu'il ne la lise pas, davantage qu'il la baragouine, dirait-on. Pour l'arabe, c'est tout l'inverse. Sa pratique se limite au début à l'espace d'une pièce : à l'école primaire, il en apprend quelques bases à partir du Coran ; chez lui, avec son grand-père, il répète ses leçons et entraperçoit déjà d'autres horizons de la culture arabe. Puis, sa pratique s'élargit à d'autres espaces de sociabilité : à la mosquée, avec le mufti de Lofça, il s'initie aux 'sciences arabes'; à la mosquée, au café ou même dans la rue, il fait partie d'un groupe de jeunes à qui un lettré de la bourgade enseigne l'exégèse coranique. Voici pour le premier âge de la carrière linguistique, de la compétence : Ahmed Cevdet connaît trois langues: une langue maternelle, une langue locale, une langue de culture.

[12] Le second, je le ferais débuter vers l'âge de dix-sept ans. C'est alors que le jeune provincial monte à la capitale pour y faire ses études. À la medrese, sa compétence s'approfondit : Ahmed continue de lire, d'écrire, de parler turc et développe son arabe, parcourant tous les domaines de son enseignement en terre ottomano-turque. Elle se redéploie aussi : à l'âge de vingt ans, il s'initie à une langue quasi-nouvelle pour lui, le persan. C'est vers l'apprentissage de celle-ci que porte alors l'essentiel de ses efforts. II ne peut l'apprendre aussi bien dans son milieu scolaire que l'arabe ou le turc : à l'en croire, elle n'est pas intégrée au curriculum des medrese. II l'apprend au 
Citation : Bouquet, Olivier (2007) 'Ce que dire que l'on parle veut dire : réflexion sur les compétences linguistiques des derniers Ottomans', European Journal of Turkish Studies, Thematic Issue $\mathrm{N}^{\circ} 6$, III-literate Knowledge, URL: http://www.ejts.org/document1523.html

To quote a passage, use paragraph (§).

contact d'un maître, dans un cercle de lettrés, dans le tekke de Murad Molla, puis dans le konak de Fehim Efendi. C'est là qu'il s'initie à la littérature mesnevi. Entre vingt et trente ans, Ahmed Cevdet devient un persanisant comme il en est bien peu. Et puis advient la dernière langue, qu'il apprend à partir de l'âge de trente ans : le français. Au début, il n'en connaît rien. II n'a pas eu l'avantage de faire un séjour en France ou en Europe. C'est à la suite de sa rencontre avec Mustafa Reşid qu'il décide de s'y initier, en secret : pour le religieux qu'il est, la langue des infidèles est proscrite.

[13] À considérer ainsi plusieurs étapes, j'oppose deux états linguistiques que la fiche biographique ne distingue pas. À l'âge de dix-sept ans, Cevdet est plus que 'familier' du bulgare : il est au sommet de sa connaissance, mais surtout de sa pratique. Au moment de l'enregistrement de la fiche, il a près de la soixantaine ; il connaît plus mal cette langue qu'il ne l'a jamais connue ; il n'est retourné qu'à quelques reprises en Bulgarie; il n'a pas trouvé l'occasion de la pratiquer au cours de sa carrière. Pour le français, c'est l'inverse ; il en ignorait tout à l'âge de dix-sept ans; il la connaît mieux qu'il ne l'a jamais connue. Il a été amené à la lire beaucoup - des classiques, surtout des ouvrages de droit - pour constituer le code civil ottoman. Aussi l'histoire de ces langues constitue-telle pour Ahmed Cevdet celle de deux courbes inverses, davantage que celle d'une égalité dont rendent habituellement compte les notices des dictionnaires biographiques. Par conséquent, loin de prendre les données des fiches biographiques pour argent comptant, l'historien doit à la fois les compléter et les réinterpréter. Quand il y trouve assez d'indices qui coïncident avec d'autres sources et suggèrent que le pacha parle ou écrit une langue dont il ne fait pas mention, ou à l'inverse ne parle pas la langue qu'il cite, il doit modifier les couleurs de son portrait biographique. Encore lui faut-il être certain de bien comprendre le sens des catégories employées, notamment celles qui recouvrent l'écrire et le parler.

\section{IV. Écrire et (ou) parler}

[14] Pour ce faire, prenons le cas d'un autre pacha, Abdülkadir :

\begin{tabular}{|l|l|l|l|}
\hline & Turc & Kurde & Persan \\
\hline Abdülkadir & Connaît & Connaît & Connaît \\
\hline
\end{tabular}


Citation : Bouquet, Olivier (2007) 'Ce que dire que l'on parle veut dire : réflexion sur les compétences linguistiques des derniers Ottomans', European Journal of Turkish Studies, Thematic Issue $\mathrm{N}^{\circ} 6$, III-literate Knowledge, URL: http://www.ejts.org/document1523.html

To quote a passage, use paragraph (§).

Celui-ci fait usage d'un terme (bilir) qui ne dit rien de ses aptitudes pratiques. Imaginons à présent qu'il est en lice pour un poste en Syrie, en concurrence avec les trois pachas précités. Si le critère linguistique intervient dans les nominations, toutes choses égales par ailleurs, le classement est le suivant :

1/ Ahmed İzzet ; Hüseyin Rüşdü

2/ Hüseyin Şukrü

3/ Abdülkadir

[15] Supposons que le poste est situé dans l'Est anatolien et que la langue prise en compte est le kurde. Le classement est alors inversé :

\section{1/ Abdülkadir}

2/ Ahmed İzzet ; Hüseyin Rüşdü ; Hüseyin Şükrü

[16] Mais alors que, dans le premier cas, l'indication linguistique garantit la bonne décision (Ahmed İzzet et Hüseyin Rüşdü disent tous deux un degré de pratique qui implique une capacité à employer l'arabe dans leurs fonctions), dans le second elle ne l'assure pas (rien ne dit qu'Abdülkadir puisse faire usage du kurde). J'écarte par conséquent les indications données par Abdülkadir : pas plus en turc qu'en français, la mention d'un 'savoir' ne permet de déduire un quelconque mode de pratique. Celle d'un savoir-faire permet en revanche d'aller plus loin.

\begin{tabular}{|l|l|l|l|}
\hline & Turc & Arabe & Persan \\
\hline Ahmed İzzet & Parle et écrit & Parle et écrit & Parle et écrit \\
\hline $\begin{array}{l}\text { Hüseyin } \\
\text { Rüşdü }\end{array}$ & Écrit & Parle & Parle \\
\hline $\begin{array}{l}\text { Hüseyin } \\
\text { Şükrü }\end{array}$ & Écrit & Familier & Familier \\
\hline
\end{tabular}

[17] Les termes kitabet et tekellüm désignent a priori l'écrit et le parler. II manque le lire, à moins qu'il ne soit implicite.

1/ C'est certainement le cas d'Ahmed İzzet : comment pourrait-il écrire une langue sans la lire, alors même que l'inverse est possible? En comparaison, si Abdüllatif Subhi mentionne un kitabet pour l'arabe et le persan et un kıraat (lire) pour le français et le grec, c'est qu'il lit les premiers et n'écrit pas les seconds. 
Citation : Bouquet, Olivier (2007) 'Ce que dire que l'on parle veut dire : réflexion sur les compétences linguistiques des derniers Ottomans', European Journal of Turkish Studies, Thematic Issue $\mathrm{N}^{\circ} 6$, III-literate Knowledge, URL: http://www.ejts.org/document1523.html

To quote a passage, use paragraph (§).

2/ Selon le même raisonnement, Hüseyin Rüşdü lit le turc, mais rien n'assure qu'il lise l'arabe et le persan. II a pu apprendre à les parler sans les lire, lors de missions dans des territoires arabophones ou persanophones.

3/ Pour la même raison, Hüseyin Şükrü lit probablement le turc. Pour les autres langues, il est nécessaire de consulter sa notice biographique dans le détail. On y lit qu'il a obtenu un diplôme de medrese. Il est donc possible qu'il lise le persan et l'arabe.

Le tableau doit donc être complété :

\begin{tabular}{|l|l|l|l|}
\hline & Turc & Arabe & Persan \\
\hline Ahmed İzzet & Parle, écrit et lit & Parle, écrit et lit & Parle, écrit et lit \\
\hline Hüseyin Rüşdü & Écrit et lit & Parle et lit & Parle \\
\hline Hüseyin Şükrü & Écrit et lit & Est familier et lit & Est familier et lit \\
\hline
\end{tabular}

[18] À partir de ces cas, quelques propositions valables pour l'ensemble des notices biographiques des sicill-i ahvâl peuvent être suggérées :

1/ Une mention kitabet doit aussi être comprise comme un savoir lire: dans ce sens, elle est l'équivalent d'une mention okur ve yazar (lire et écrire). Elle se rapproche de la notion anglo-saxonne de literacy.

2/ Une mention tekellüm ne doit être comprise comme un savoir lire que dès lors qu'un élément de la notice le confirme.

[19] Reste à déterminer les modalités du recouvrement de l'écrit et du parler. On remarque d'abord que la notion d'écriture est souvent inclusive d'une capacité orale. Le savoir écrit (mentionné) désigne un savoir parler (non mentionné), au moins dans les cas de figure suivants :

1/ La même chose est dite pour chaque langue, y compris dans le fait qu'aucune n'est présentée comme parlée : à moins de considérer qu'Ahmed İzzet ne cite pas la langue qu'il parle, force est de penser qu'il parle au moins l'une de celles qu'il cite : né à Kastamonu, Ahmed İzzet a fait ses études à Isparta. Dans des zones turcophones de l'Empire, donc.

2/ Une seule langue est citée : 'il écrit le turc'. Pour les mêmes raisons, je déduis que cette seule langue est celle que le pacha parle. Dès lors, cette indication a exactement le même sens qu'une autre tout aussi fréquente : 'il écrit et parle le turc'. 
Citation : Bouquet, Olivier (2007) 'Ce que dire que l'on parle veut dire : réflexion sur les compétences linguistiques des derniers Ottomans', European Journal of Turkish Studies, Thematic Issue $\mathrm{N}^{\circ} 6$, III-literate Knowledge, URL: http://www.ejts.org/document1523.html To quote a passage, use paragraph (§).

3/ La notion de kitabet est juxtaposée à la notion de lire et d'écrire: Ali Muhiddin et Mahmud Celâleddin, tous deux turcophones, citent un kitabet pour le turc et un okur yazar pour le français. La différence, en plus d'inclure une nuance de compétence (literacy), peut aussi renvoyer à une différence de savoir parler. Autrement dit, le kitabet indique plus une compétence développée, notamment orale, qu'il ne désigne une seule aptitude à l'écrit.

4/ Aucune langue n'est dite parlée alors que le discours est différencié.

\begin{tabular}{|l|l|l|l|}
\hline & Écrit & Lit & Familier \\
\hline Hüseyin & Turc & Français, grec, arménien & Géorgien \\
\hline
\end{tabular}

[20] Selon l'hypothèse que la langue écrite est celle qu'il connaît le mieux, Hüseyin parle nécessairement le turc. Mais il est impossible de savoir s'il parle les autres langues. Dans les autres cas, kitabet doit être entendu comme seule maîtrise scripturaire, surtout lorsque rien dans la fiche ne vient signaler le contraire : Hüseyin Rüşdü, d'une grande famille d'Alep, donc arabophone, ne laisse en rien croire qu'il parle le turc (nulle précision d'étude du turc, aucune fonction en terre turcophone).

[21] Inversement, la notion d'oralité ne recouvre celle d'écriture qu'exceptionnellement, comme dans les cas suivants :

1/ Une indication de savoir écrire est indiquée pour une autre langue : Ahmed Necib écrit (kitabet) le turc ; donc, en vertu de l'induction précédente, il le parle ; il parle le français ; donc, il ne l'écrit pas.

2/ Le caractère limité du savoir parler est explicite: Mustafa Nuri écrit et parle le turc, l'arabe, le persan, le français et le grec ; il parle 'seulement' l'italien et l'albanais.

3/ La langue signalée n'est pas une langue véhiculée par l'écrit: l'albanais par exemple que personne ne dit savoir écrire dans les fiches.

4/ Une indication de la fiche confirme un savoir parler exclusif : Ahmed Kemal mentionne un tekellüm pour l'arabe ; or il est révoqué pour ne pas savoir lire la presse arabe locale.

[22] Au total, on retiendra les points suivants :

1/ Une indication kitabet isolée pour une langue inclut un savoir parler. Elle doit donc être distinguée de l'indication yazar. 
Citation : Bouquet, Olivier (2007) 'Ce que dire que l'on parle veut dire : réflexion sur les compétences linguistiques des derniers Ottomans', European Journal of Turkish Studies, Thematic Issue $\mathrm{N}^{\circ} 6$, III-literate Knowledge, URL: http://www.ejts.org/document1523.html

To quote a passage, use paragraph (§).

2/ Une indication kitabet isolée pour une langue associée à des indications tekellüm pour d'autres langues peut aussi bien indiquer un savoir parler supplémentaire qu'un seul savoir écrire (au sens de yazar).

3/ Une indication tekellüm n'indique presque jamais un savoir écrire. En revanche, elle peut renvoyer à un savoir lire.

\section{Une affaire de compétence}

[23] Différenciées, les indications des notices biographiques appellent un traitement différencié. C'est ce que négligent les dictionnaires biographiques qui mettent sur un même plan la connaissance de chaque langue chez un individu. Ainsi Y. Öztuna dit d'Abdüllatif Subhi qu'il connaît l'arabe, le persan, le français, le grec, le latin et l'hébreu. Or sa fiche ne dit rien du latin ou de l'hébreu, ce qui peut laisser entendre qu'il connaît moins bien ces langues que les autres. L'homme n'est pas plus un polyglotte parfait qu'il n'est un touche-à-tout ; c'est un véritable lettré qui connaît certes plusieurs langues, mais les manipule différemment. Penser autrement, c'est négliger ce qu'on appelle en linguistique la compétence, dont rend compte la fiche d'Abdüllatif Subhi. C'est également négliger des niveaux de maîtrise différents pour chaque langue. Pour essayer de les reconstituer, je voudrais considérer cinq indicateurs :

[24] 1/ La mention d'une connaissance

\begin{tabular}{|l|l|l|l|}
\hline & Turc & Kurde & Persan \\
\hline Abdülkadir & Connaît & Connaît & Connaît \\
\hline
\end{tabular}

Parmi ces langues citées, certaines sont mieux connues que d'autres. Abdülkadir est né à Kirkuk (ville peuplée de Kurdes) et y a fait ses études; il est probable que le kurde soit sa langue maternelle, une langue très peu écrite alors. En tant qu'agent de l'État, il est censé écrire, lire et parler le turc. Mais rien n'assure que dans aucun de ces domaines son niveau soit élevé : rien dans ses études n'indique un apprentissage du turc; sa carrière s'est déroulée dans des régions kurdophones ou arabophones. Cela dit, comme il ne fait aucune mention de l'arabe, on peut donc imaginer qu'au moins dans les secondes, c'est le turc qu'il utilise dans ses fonctions, comme langue 
Citation : Bouquet, Olivier (2007) 'Ce que dire que l'on parle veut dire : réflexion sur les compétences linguistiques des derniers Ottomans', European Journal of Turkish Studies, Thematic Issue $\mathrm{N}^{\circ} 6$, III-literate Knowledge, URL: http://www.ejts.org/document1523.html

To quote a passage, use paragraph (§).

de travail et de communication avec sa hiérarchie. Enfin, pour beaucoup de Kurdes, le persan sert de langue écrite. Peut-être Abdülkadir le parle-t-il également. Mais rien ne peut être dit de son niveau.

[25] On peut certes transformer ces données de connaissance en données de pratique:

\begin{tabular}{|l|l|l|l|}
\hline & Turc & Kurde & Persan \\
\hline Abdülkadir & Écrit et parle & Parle & Écrit (parle ?) \\
\hline
\end{tabular}

Mais, il est difficile d'indiquer un niveau particulier.

[26] 2/ La mention d'une pratique

Reprenons les données complétées pour Ahmed İzzet:

\begin{tabular}{|l|l|l|l|}
\hline & Turc & Arabe & Persan \\
\hline Ahmed İzzet & Parle, écrit et lit & Parle, écrit et lit & Parle, écrit et lit \\
\hline
\end{tabular}

Les langues sont mises sur le même plan. Pourtant, même chez les dignitaires les plus renommés pour leur art oratoire ou scripturaire, certains parlent bien mieux qu'ils n'écrivent, et inversement. Ici, on peut opter pour l'hypothèse d'une corrélation: plus les usages individuels d'une langue sont diversifiés (c'est ce que les anglo-saxons entendent par versatility), plus est élevé le niveau de chacun de ces usages. À moins de considérer un phénomène d'exclusion : Ahmed Arifi montre une grande aptitude à écrire le français - surtout lorsqu'il s'agit d'affaires politiques -, mais il ne le parle pas. Dans l'incapacité de trancher, il faut se reporter à la biographie du dignitaire. Le turc: Ahmed İzzet n'est pas né et n'a pas fait ses études dans une région turcophone de l'Empire, mais il a été en poste à Konya comme secrétaire en chef de la direction des contributions indirectes, poste qui exige une maîtrise de l'écriture turque. L'arabe : c'est très probablement la langue dont il a la plus grande maîtrise : il est né à Musul ; il indique avoir étudié les sciences arabes au contact de maîtres; il a écrit un ouvrage en arabe ; il a mené l'essentiel de sa carrière dans le monde arabe. Le persan : il ne dit pas l'avoir étudié, mais semble avoir été consul en Iran. Je serais donc tenté de proposer la hiérarchie suivante : arabe, turc, persan.

\section{[27] 3/ Le souci de précision}

De la même façon que l'employé décrit les espaces de la pratique au-delà des exigences de l'administration, il sort des limites du cadre lexical imposé pour rendre compte du niveau de sa 
Citation : Bouquet, Olivier (2007) 'Ce que dire que l'on parle veut dire : réflexion sur les compétences linguistiques des derniers Ottomans', European Journal of Turkish Studies, Thematic Issue $\mathrm{N}^{\circ} 6$, III-literate Knowledge, URL: http://www.ejts.org/document1523.html

To quote a passage, use paragraph (§).

maîtrise. Tantôt il le minore : il dit parler, écrire, du moins 'un peu'; il dit en être familier. Tantôt il le majore: Mehmed Said parle et écrit le turc 'extrêmement bien'. D'autres disent écrire ou (et) parler une langue avec facilité, se targuent d'avoir une belle plume, ou précisent que, comme il s'agit de leur langue maternelle, ils la savent 'naturellement'. Ce faisant, tous instituent une double hiérarchie de niveau, individuellement (entre une langue et d'autres qu'ils disent parler et écrire) et collectivement (entre la plupart des employés qui manient cette langue et eux-mêmes). Rendre précis, c'est se distinguer. Tantôt enfin le pacha différencie sa compétence : il souligne que s'il écrit ou parle plusieurs langues, les degrés de sa maîtrise varient de l'une à l'autre.

\section{[28] 4/ Les informations des notices}

Elles sont plus ou moins éclairantes :

- la scolarité, moment d'apprentissage principal, en tout cas premier, des langues : la mention de 'familiarité' de français par Abdullah Galib fait écho à la précision qu'il donne juste avant ('il parle un peu le français').

- la publication d'ouvrages.

- l'enseignement des langues.

- les indications de séjour dans la rubrique de la carrière : Ibrahim Edhem n'indique pas ses niveaux de langue. Mais il a passé une dizaine d'années en France dans sa jeunesse.

- les indications du supérieur hiérarchique : Ahmed Rasım Paşa, vali de Yanya (Janina), certifie 'la compétence et le talent' de son subordonné, Timur Paşa, dans les langues qu'il cite dans sa fiche ; Osman Paşa signale les capacités en arabe et persan de Hüseyin Şukrü.

\section{[29] $\underline{5 / \text { Les autres sources }}$}

II est tout aussi précieux de faire appel aux sources complémentaires: participation à des conférences internationales, rédaction d'essais et d'articles, traduction d'ouvrages, description d'un pacha comme connaisseur de langues sont autant d'indicateurs qui permettent d'affiner l'évaluation de niveau. Mais ces informations ne concernent que les pachas répertoriés, les plus renommés. Pour les autres, il ne faut compter qu'avec la fiche biographique. Fort heureusement, s'il est difficile d'inférer à partir d'une mention de pratique isolée un degré de maîtrise, il est généralement plus aisé de déterminer pour chaque pacha la hiérarchie des langues pratiquées. 
Citation : Bouquet, Olivier (2007) 'Ce que dire que l'on parle veut dire : réflexion sur les compétences linguistiques des derniers Ottomans', European Journal of Turkish Studies, Thematic Issue $\mathrm{N}^{\circ} 6$, III-literate Knowledge, URL: http://www.ejts.org/document1523.html

To quote a passage, use paragraph (§).

\section{Conclusion}

[30] L'historiographie ottomaniste utilise souvent la connaissance des langues comme un double critère d'évolution historique. Un critère de déclin : si les medrese ne sont plus au 19ième siècle les hauts lieux de savoir qu'elles auraient été à 'l'époque classique', c'est aussi qu'on y apprend moins bien l'arabe et le persan. Un critère de modernité : si les élites ont accès aux arts et aux sciences venus d'ailleurs, c'est qu'ils apprennent davantage ou mieux les langues occidentales, le français en particulier. Les remarques précédentes ne remettent pas en question cette double thématique. En revanche, elles invitent à considérer la connaissance des langues comme des pratiques plus que comme des savoirs; des savoirs pratiqués en somme. Du coup, le portrait linguistique est bien moins flatteur. Car ceux qui passent pour des lettrés, au sens de savants ou d'érudits, ou qui cherchent à se faire passer comme tels, n'ont souvent qu'une compétence réduite, au sens que les linguistes prêtent à ce terme. Pour reprendre les termes du dossier, literacy n'est pas versatility; et ce n'est pas parce que tel employé dit qu'il sait l'arabe, qu'il sait l'écrire ou le parler. De même, ce n'est pas parce qu'il dit savoir l'écrire, qu'il ne peut pas le parler. Toute donnée linguistique isolée prélevée dans telle ou telle source doit être rapportée à l'ensemble des termes du discours autobiographique, et autant que faire se peut, à d'autres sources: Ahmed Pacha dit qu'il écrit l'arabe, mais ne le parle-t-il pas également ? L'écrit-il aussi bien qu'il le laisse entendre ? L'écritil mieux que Mehmed Pacha qui certes n'évoque pas cette langue, mais est réputé pour la manier avec aisance ? On a souvent dit ou écrit qu'au pays du sultan où se parlaient tant de langues, peu étaient ceux qui savaient les lire ou les écrire. L'affaire se complique ; la ligne tracée entre culture orale et culture écrite se brouille : d'un côté, les illettrés ne sont pas les ignorants que l'on croit dès lors qu'on les envisage comme des hommes de langue(s); de l'autre, bien des réputations d'érudits ou de polyglottes se défont si l'on considère le champ des compétences linguistiques sous toutes ses formes. 
Citation : Bouquet, Olivier (2007) 'Ce que dire que l'on parle veut dire : réflexion sur les compétences linguistiques des derniers Ottomans', European Journal of Turkish Studies, Thematic Issue $\mathrm{N}^{\circ} 6$, III-literate Knowledge, URL: http://www.ejts.org/document1523.html

To quote a passage, use paragraph (§).

\section{References}

Albayrak, Sadık (1980-1981) Son Devir Osmanlı Ulemâsı (IImiye Ricâlının Teracim-i Ahvâli), 4 Vol., Istanbul, İstanbul Büyükşehir Belediyesi.

Bouquet, Olivier (2007) Les Pachas du sultan. Essai sur les agents supérieurs de l'État ottoman (1839-1909), Louvain, Peeters.

Bourdieu, Pierre (1982) Ce que parler veut dire. L'économie des échanges linguistiques, Paris, Fayard.

Cevdet Paşa (1991), Tezâkir 40, Cavid Baysun (éd.), Ankara, Türk Tarih Kurumu (3e éd.).

Clayer, Nathalie (2000) 'Les Cadis de l'Après Tanzimat : l'exemple des cadis originaires d'Ergiri et Libohova', Turcica 32, pp. 33-58.

Çankaya, Ali M. (1968-1969) Yeni Mülkiye Tarihi ve Mülkiyeliler, 8 Vol., Ankara.

Danişmend, İsmail Hamdi (1971-1972) İzahlı Osmanlı Tarihi Kronolojisi, 5 Vol., Istanbul, Türkiye Basımevi.

Findley, Carter V. (1989) Ottoman Civil Officialdom. A Social History, Princeton, Princeton University Press.

Georgeon, François (2003) Abdülhamid II. Le sultan calife (1876-1909), Paris, Fayard.

Gövsa, İrahim Alâettin (1946) Türk Meşhurları Ansiklopedisi, Istanbul, Yedigün, 1946.

Gross, Max (1979) 'Ottoman Rule in the Province of Damascus, 1860-1909', Unpub. Ph.D. Diss., Georgetown University.

İnal, İbnülemin Mahmud Kemal (n.d.) Osmanlı Devrinde Son Sadrazamlar, 4 Vol., Istanbul.

İnal, İbnülemin Mahmud Kemal (1969) Son Asır Türk Şairleri, 4 Vol., Istanbul, (rééd.).

Kélékian, Diran (1911) Dictionnaire turc-français, Istanbul, Mihran.

Kiel, Machiel (1990) 'Urban development in Bulgaria in the Turkish period: the place of Turkish architecture in the process' in Karpat, Kemal (ed.), The Turks of Bulgaria: the History, Culture and Political Fate of a Minority, Istanbul, Isis, pp. 79-158.

Malumat 2 (37), 1312.

Mardin, Ebul'ülâ (1946) Medenî Hukuk Cephesinden: Ahmet Cevdet Paşa, 1822-1895, Istanbul, İ. Ü. Hukuk Fakültesi.

Mercan, Mehmet 'Sadriazam Ahmed Cevad Paşa', thèse de doctorat, Université de Marmara, Institut des Sciences sociales, Istanbul, 1998.

Meriç, Ümid (1979) Cevdet Paşa'nın Cemiyet ve Devlet Görüşü, İstanbul, Ötüken (2e éd.).

Özcan, Azmi (1999) 'Ahmed Cevad Paşa', in Yaşamları ve Yapıtlarılla Osmanlılar Ansiklopedisi, 2 Vol., Istanbul, Yapı Kredi Yayınları, Vol. 1, pp. 112-113. 
Citation : Bouquet, Olivier (2007) 'Ce que dire que l'on parle veut dire : réflexion sur les compétences linguistiques des derniers Ottomans', European Journal of Turkish Studies, Thematic Issue $\mathrm{N}^{\circ} 6$, III-literate Knowledge, URL: http://www.ejts.org/document1523.html

To quote a passage, use paragraph (§).

Öztuna, Yılmaz (1996), Devletler ve Hânedanlar. Türkiye (1074-1990), Ankara, Kültür Bakanlığı Yay. (2e éd.).

Redhouse Türkçe-Ingilizce Sözlük (1997) Istanbul, SEV (16e éd.).

Sicill-i ahvâl defterleri, Archives de la Présidence du Conseil, Istanbul (SA).

Strauss, Johann (1995) 'Diglossie dans le domaine ottoman. Évolution et péripéties d'une situation linguistique', Revue du monde musulman et de la Méditerranée 75-76, pp. 221-255.

Süreyya, Mehmed (1891-1893) Sicill-i Osmani Yahud Tezkire-i Meşahir-i Osmaniye, 4 Vol., Istanbul, Matbaa-i amire.

Szyliowicz, Joseph S. (1975) 'Changes in the Recruitment Patterns and Career-lines of Ottoman Provincial Administrators during the Nineteenth Century', in Ma'oz, Moshe (ed.), Studies on Palestine during the Ottoman Period, Jerusalem, The Magnes Press, pp. 249-283. 